\title{
RANKL, OPG and CTR mRNA expression in the temporomandibular joint in rheumatoid arthritis
}

\author{
WEI-WEI LIU ${ }^{1}$, ZHI-MIN XU ${ }^{1}$, ZHENG-QIANG LI ${ }^{1}$, YAN ZHANG ${ }^{2}$ and BING HAN ${ }^{1}$ \\ ${ }^{1}$ Department of Oral and Maxillofacial Surgery, School of Stomatology, Jilin University; \\ ${ }^{2}$ Department of General Therapy Dentistry, School of Stomatology, Jilin University, Changchun, Jilin 130021, P.R. China
}

Received August 28, 2014; Accepted June 11, 2015

DOI: $10.3892 /$ etm.2015.2629

\begin{abstract}
The calcitonin receptor (CTR) and receptor activator of nuclear factor $\kappa \mathrm{B}$ ligand (RANKL) have been found to be involved in the differentiation of osteoclasts. The association between the RANKL:osteoprotegerin (OPG) expression ratio and the pathogenesis of bone-destructive rheumatoid arthritis (RA) has been described in several joints, but the available data for the temporomandibular joint (TMJ) are limited. The aim of the present study was to investigate the involvement of osteoclasts at sites of bone erosion by determining the CTR expression and the RANKL:OPG expression ratio in the TMJ in a collagen-induced arthritis (CIA) model. Forty-eight male Wistar rats were randomly divided into two groups: Control group, injected with saline solution for 6 weeks; and CIA group, injected with emulsion. The RANKL and OPG mRNA expression was significantly increased in immunized rats compared with that in non-immunized rats. The RANKL:OPG expression ratio on the trabecular bone surface was 9.0 and 6.4 in the CIA group at weeks 4 and 6, respectively, while the RANKL:OPG expression ratio in the controls was 1.0:2. CTR mRNA expression was significantly upregulated in immunized rats compared with that in non-immunized rats; the level of CTR mRNA in the CTR-positive osteoclasts on the trabecular bone surface was 10.9- and 7.8-fold higher in the CIA rats than that in the control rats at weeks 4 and 6, respectively. In conclusion, focal bone destruction in an experimental model of arthritis in the TMJ can be attributed to cells expressing CTR, a defining feature of osteoclasts. The expression of RANKL and OPG mRNA within the inflamed synovium provides an insight into the mechanism of osteoclast differentiation and function at the border of bone erosion in arthritis.
\end{abstract}

Correspondence to: Dr Bing Han, Department of Oral and Maxillofacial Surgery, School of Stomatology, Jilin University, 1500 Qinghua Road, Changchun, Jilin 130021, P.R. China

E-mail: hbing@jlu.edu.cn; 444383490@qq.com

Key words: calcitonin receptor, osteoprotegerin, rheumatoid arthritis, receptor activator of nuclear factor $\mathrm{\kappa B}$ ligand, focal bone destruction, bone erosion, inflamed synovium, experimental arthritis

\section{Introduction}

Rheumatoid arthritis (RA) is a chronic systemic disease that leads to inflammation and tissue damage in joints and is characterized by symmetrical joint alteration; however, the etiology of the condition has yet to be fully elucidated. The temporomandibular joint (TMJ) is known to be frequently affected by RA $(1,2)$, with reported frequencies of TMJ involvement varying between 2 and $86 \%(2,3)$. TMJs afflicted with RA may be associated with pain, swelling, crepitation, stiffness on opening the mouth and limitation of movement (4). The initial changes that can be observed in the degenerating TMJ are proteoglycan degradation and softening in the fibrocartilage of the condylar and articular eminence (5). Severe destruction of the cortical and subcortical bone can ultimately lead to almost complete loss of the condyle. These changes are followed by the exposure of subchondral bone and bone resorption by osteoclasts (6).

Osteoclasts are multinucleated cells responsible for bone resorption. The identification and quantification of osteoclasts, as well as their development in osteoclastogenic clusters and bone, require cell markers that are specific to this type of cell; therefore, tartrate-resistant acid phosphatase (TRAP), the vitronectin receptor (VNR) and the calcitonin receptor (CTR) are frequently utilized in osteoclast identification (7). The expression of TRAP and VNR, however, can be observed prior to the expression of CTR and indicates the presence of cells at an intermediate stage of differentiation (8). Furthermore, TRAP and VNR are expressed by macrophages, which can cause complications in long-term bone marrow cultures in which both osteoclasts and macrophage polykaryons are formed. As a result of the issues associated with these phenotypic markers, the determination of CTR expression is currently considered to be the only approach suitable for providing both proof of the presence of osteoclasts and the quantitative data associated with these osteoclasts (9). Furthermore, the identification of mononuclear osteoclasts is, at present, only possible through the detection of cells expressing CTR (8). Although the CTR is known to play a role in bone resorption in other joints (10), the importance of its role in the TMJ arthritis model has not yet been elucidated.

Bone remodeling is one of the main metabolic activities necessary for maintaining the normal structure and function of bones. Osteoclasts play an important role in these processes; 
osteoprotegerin (OPG), receptor activator of nuclear factor- $\kappa \mathrm{B}$ (RANK), and RANK ligand (RANKL) co-regulate the functions of osteoclasts. Osteoclastic bone destruction is induced by RANKL, while OPG acts as a decoy receptor and prevents RANKL binding to RANK, its receptor, thus limiting bone destruction. The activity of osteoclasts is likely to depend, at least partially, on the relative balance existing between RANKL and OPG expression, and studies have suggested the involvement of RANKL and OPG in the pathogenesis of RA (11,12). An increase in the RANKL:OPG expression ratio in the microenvironment of a joint with RA suggests a major role of the RANKL-OPG pathway in RA pathogenesis, as it has the potential to induce osteoclastogenesis (13).

The RANKL:OPG expression ratio in the local bone microenvironment determines the direction of osteoclast differentiation. Only CTR expression unambiguously identifies osteoclasts and distinguishes them from macrophage polykaryons. Several studies have described the involvement of the CTR and the RANKL:OPG expression ratio in the pathogenesis of bone-destructive RA in other joints (14-16), but the available data for their role in the TMJ are limited. The aim of the present study, therefore, was to investigate the involvement of osteoclasts at sites of bone erosion using the osteoclast-specific marker CTR expression and to determine the RANKL:OPG expression ratio in the TMJ.

\section{Materials and methods}

Animals. A total of 48 male Wistar rats (Laboratory Animal Center of Jilin University, Changchun, China) aged $\sim 5$ weeks and weighing 105-135 g, were included in this study. All experimental procedures complied with the requirements of the Animal Care and Use Committee of Laboratory Animal Center of Jilin University. All rats were allowed to adapt to the facilities for one week and were then randomly separated into two groups with 24 individuals: i) Control group (healthy subjects), and ii) collagen-induced arthritis (CIA) group.

Induction of CIA. The induction of arthritis was performed as previously described (17). Briefly, the CIA model was established by intradermally injecting the rats at the base of the TMJ with $100 \mu \mathrm{l}$ of an emulsion that contained $100 \mu \mathrm{g}$ bovine type II collagen ( $2 \mathrm{mg} / \mathrm{ml}$; Sigma-Aldrich, St. Louis, MO, USA) dissolved in $0.05 \mathrm{M}$ acetic acid and an equal volume of complete Freund's adjuvant. Following confirmation of the establishment of mononuclear cell infiltration, synovial lining and villous hyperplasia and pannus formation, the unilateral intra-articular injection of emulsion into the TMJ was performed. Saline was injected into the contralateral joint as sham induction.

Hematoxylin and eosin (HE) staining. The animals were sacrificed using an overdose of sodium pentobarbital (Nembutal ${ }^{\circledR}$; Abbott Laboratories, Abbott Park, IL, USA). Six rats were sacrificed at 0,2, 4 and 6 weeks after the induction of arthritis. Bilateral samples of the TMJ were collected, fixed in $4 \%$ paraformaldehyde for $24 \mathrm{~h}$, decalcified with EDTA solution (10\%) and embedded in paraffin. Sagittal sections measuring $4 \mathrm{~mm}$ were cut in series, and HE was used to stain every 10th section. Adjacent sections to those used for HE staining were used for immunohistochemical analysis. Endogenous peroxidase activity was terminated using $3 \% \mathrm{H}_{2} \mathrm{O}_{2}$ in methanol, and the sections were then incubated overnight with primary antibodies: $10 \mathrm{mg} / \mathrm{ml}$ goat anti-rabbit interleukin (IL)- $1 \beta$ or $5 \mathrm{mg} / \mathrm{ml}$ sheep anti-rabbit IL-1 receptor antagonist at $4^{\circ} \mathrm{C}$ (18). For the negative control, normal rabbit IgG (CST Biological Reagents Company Ltd., Shanghai, China) was used as the primary antibody. Following incubation with the primary antibody, the sections were incubated for $30 \mathrm{~min}$ in $5 \mathrm{mg} / \mathrm{ml}$ biotinylated rabbit anti-goat or anti-sheep IgG (Vector Laboratories, Burlingame, CA, USA) and then for a further $30 \mathrm{~min}$ using an Elite $\mathrm{ABC}^{\circledR}$ kit (Vector Laboratories). Diaminobenzidine (DAB Reagent Set; KPL, Inc., Gaithersburg, MD, USA) was used as a chromogen, and the sections were counterstained with HE. In addition, TRAP was detected in the paraffinized tissue sections using a commercial acid phosphatase leukocyte kit (Sigma-Aldrich).

Enzyme-linked immunosorbent assay (ELISA). Serum concentrations of tumor necrosis factor- $\alpha$ (TNF- $\alpha)$ and IL-1 $\beta$ were measured by ELISA as previously described (19). Blood samples were collected by cardiac puncture at $0,2,4$ and 6 weeks, and the serum concentrations of TNF- $\alpha$ and IL-1 $\beta$ were measured using rat TNF- $\alpha$ and IL-1 $\beta$ double-antibody sandwich ELISA kits (Endogen, Inc., Cambridge, MA, USA), in accordance with the manufacturer's instructions. The mean of three assays was used in the analysis.

Reverse transcription-polymerase chain reaction ( $R T-P C R)$. Total RNA was isolated from the synovial membrane of the TMJ using TRIzol ${ }^{\circledR}$ reagent (Invitrogen Life Technologies, Carlsbad, CA, USA). The RT-PCR was conducted in accordance with a previously described method (20).

The primers used in this study are listed in Table I. The PCR consisted of denaturation at $94^{\circ} \mathrm{C}$ for $1 \mathrm{~min}$ (RANKL), $95^{\circ} \mathrm{C}$ for $40 \mathrm{sec}\left(\mathrm{OPG}\right.$ and $\beta$-actin) or $94^{\circ} \mathrm{C}$ for $40 \mathrm{sec}$ (GAPDH and CTR), followed by annealing at $58^{\circ} \mathrm{C}$ for $1 \mathrm{~min}$ (RANKL), $60^{\circ} \mathrm{C}$ for $1 \mathrm{~min}(\mathrm{OPG}$ and $\mathrm{GAPDH})$ or $60^{\circ} \mathrm{C}$ for $1 \mathrm{~min}(\mathrm{CTR}$ and $\beta$-actin) for 30,28 and 30 cycles, respectively, with a final extension step at $72^{\circ} \mathrm{C}$ for $10 \mathrm{~min}$. The reproducibility of the RT-PCR results was ensured by repeating each experiment three times. A sample of PCR-amplified product $(6 \mu \mathrm{l})$ was subjected to $2 \%$ agarose gel electrophoresis for quantification, and the bands were visualized using ethidium bromide. The RANKL: $\beta$-actin, OPG: $\beta$-actin and CTR:GAPDH ratios were calculated for each lane and used to compare the mRNA expression of RANKL, OPG and CTR at different time-points.

Statistical analysis. Statistical analysis was conducted using SPSS software for Windows (version 11.5; SPSS, Inc., Chicago, IL, USA). All data are presented as the mean \pm standard error of the mean. Differences between groups were analyzed using two-way analysis of variance, and $\mathrm{P}<0.05$ was considered to indicate a statistically significant difference.

\section{Results}

Histological examination of joints with CIA. HE staining of TMJs in the CIA group showed severe synovitis, including destruction of the cartilage and bone by pannus tissue, which 
Table I. Sequences and expected fragment sizes of synthetic oligonucleotides used for the reverse transcription-polymerase chain reaction.

\begin{tabular}{llr} 
Gene & \multicolumn{1}{c}{ Primer sequence } & Product (base pairs) \\
\hline RANKL & Sense: 5'-GAGACTACGGCAAGTA-3' & 822 \\
& Antisense: 5'-CCTCCAACGTTTATGG-3' & \\
OPG & Sense: 5'-TGGAGCTCGAATTCTGCTTG-3' & 603 \\
\multirow{2}{*}{-actin } & Antisense: 5'-CATCAAGATGCGGAGCTGCT-3' \\
& Sense: 5'-GAAATCGTGCGTGACATTAAG-3' \\
CTR & Antisense: 5'-CTAGAAGCATTTGCGGTGCA-3' \\
& Sense: 5'-ACACCCTGACAGCAACCGAACCT-3' & 410 \\
GAPDH & Antisense: 5'-GAACCCCCAGCCAAGTAAATAGTA-3' & 447 \\
& Sense: 5'-TATTGGGCGCCTGGTCACCA-3' & 746 \\
& Antisense: 5'-ACTACTGTAGTTCTTCCACC-3'
\end{tabular}

RANKL, receptor activator of nuclear factor $\mathrm{kB}$ ligand; OPG, osteoprotegerin; CTR, calcitonin receptor.

Table II. Serum levels of tumor necrosis factor- $\alpha(\mathrm{pg} / \mathrm{ml})$ in condylar cartilage of control and collagen-induced joints.

\begin{tabular}{lcccc}
\hline Group & Week 0 & Week 2 & Week 4 & Week 6 \\
\hline Healthy control & $25.68 \pm 1.28$ & $26.86 \pm 2.68$ & $27.38 \pm 4.20$ & $27.16 \pm 2.78$ \\
Collagen-induced joints & $26.12 \pm 0.36$ & $29.78 \pm 1.18$ & $37.91 \pm 2.33^{\mathrm{a}}$ & $12.16 \pm 2.13^{\mathrm{a}}$ \\
\hline
\end{tabular}

${ }^{\text {aS }}$ Statistically significant difference between means of healthy control and collagen-induced joints (Mann-Whitney U-test, $\mathrm{P}<0.01$ ).

Table III. Serum levels of interleukin- $1 \beta(\mathrm{pg} / \mathrm{ml})$ in condylar cartilage of control and collagen-induced joints.

\begin{tabular}{lcccc}
\hline Group & Week 0 & Week 2 & Week 4 & Week 6 \\
\hline Healthy control & $3.69 \pm 0.46$ & $4.65 \pm 1.13$ & $4.26 \pm 0.52$ & $4.19 \pm 2.23$ \\
Collagen-induced joints & $3.76 \pm 0.34$ & $10.25 \pm 1.54^{\mathrm{a}}$ & $16.18 \pm 1.20^{\mathrm{a}}$ & $12.16 \pm 2.13^{\mathrm{a}}$ \\
\hline
\end{tabular}

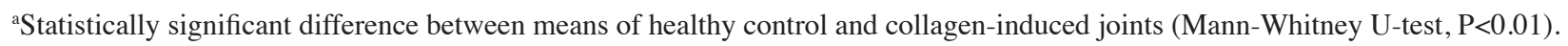

consisted of a hyperplastic synovium with fibroblast-like cells, multiple polymorphonuclear leucocytes and a limited number of mononuclear lymphocytes. Areas of severe synovitis exhibited lining cell hypertrophy and the invasion of spindle-shaped synovial cells into the stromal sublining. Furthermore, the proliferation of spindle-shaped fibroblast-like cells and inflammatory cell infiltration were noted in the pannus tissue on the articular surface, and multiple TRAP-positive, mature, multinucleated osteoclasts were observed, predominantly on the eroded surface of the bone (Figs. 1-3).

Analysis of TNF- $\alpha$ and $I L-1 \beta$. As shown in Table II, the serum levels of TNF- $\alpha$ in the collagen-induced joints were significantly increased compared with those in the control joints. The 2-week period after the induction of arthritis is considered to be the acute stage of the response. Table III shows that the serum levels of IL-1 $\beta$ in the collagen-induced joints were significantly increased compared with those in the control joints. The serum levels of IL- $1 \beta$ and TNF- $\alpha$ peaked 4 weeks after the induction of arthritis.

Expression of RANKL, OPG, and CTR $m R N A$ in rheumatoid pannus tissue. The mRNA expression of genes associated with osteoclastogenesis in the synovium was investigated using RT-PCR; the expression of the genes was confirmed by the presence of specific single bands. The results demonstrated that RANKL and OPG mRNA expression in the rat CIA model was significantly increased compared with that in the non-immunized rats (Figs. 4 and 5). The mRNA expression levels of RANKL peaked 4 weeks after immunization, and at weeks 2 and 6 were significantly higher than levels in the synovium of non-immunized rats. Mirroring the increase in the number of osteoclasts, the RANKL:OPG expression ratio on the trabecular bone surface increased to $9.0: 1$ and 6.4:1 in the CIA rats at weeks 4 and 6, respectively, while the RANKL:OPG expression ratio in the control group was 1.0:2 (Fig. 6). 


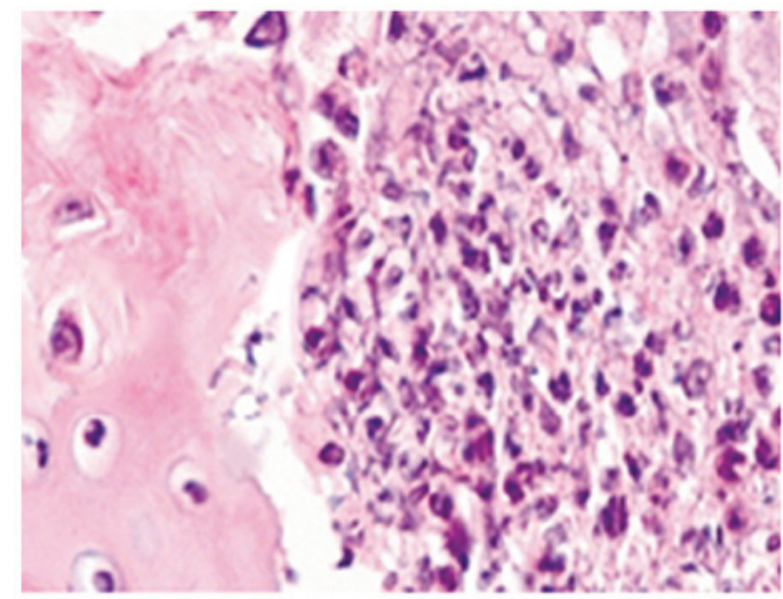

Figure 1. Histology of the arthritic temporomandibular joint (stain, hematoxylin and eosin; magnification, $x 100$ ). In the area of pannus tissue on the articular surface, the proliferation of infiltrating inflammatory cells and of spindle-shaped fibroblast-like cells could be observed, and numerous tartrate-resistant acid phosphatase-positive mature multinucleated osteoclasts were found predominantly on the eroded bone surface.

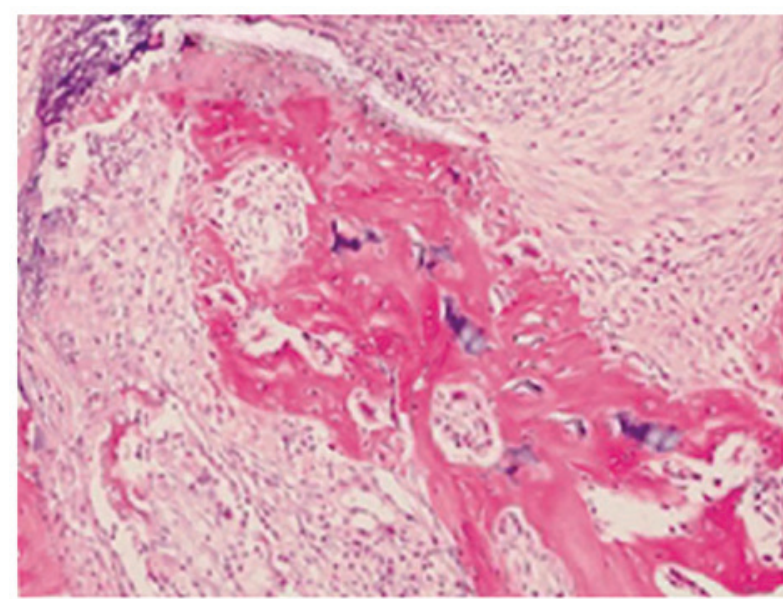

Figure 2. Histology of the arthritic temporomandibular joint (stain, hematoxylin and eosin; magnification, $x 40$ ). Inflammatory cell infiltration, with broken collagen fibers, was detected on the articular surface of the condyle.

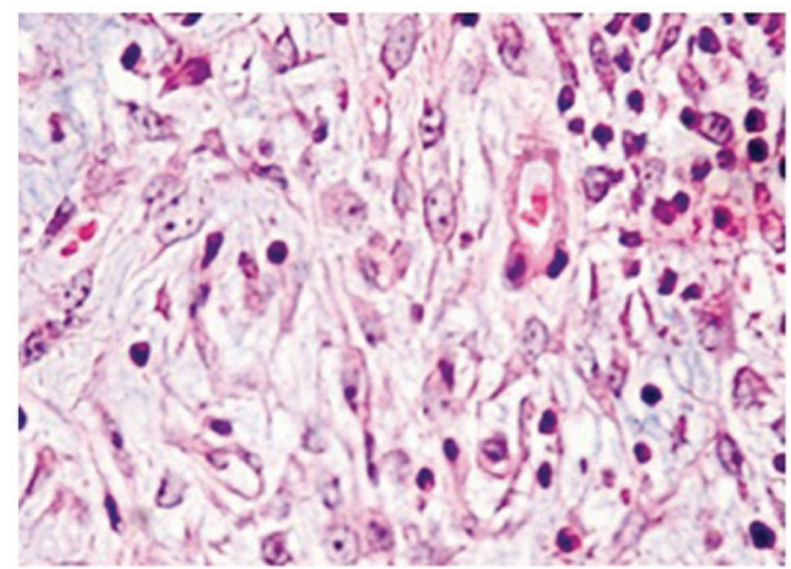

Figure 3. Histology of the arthritic temporomandibular joint (stain, hematoxylin and eosin; magnification, x300). Inflammatory cell infiltration, with broken collagen fibers, was detected on the articular surface of the condyle.

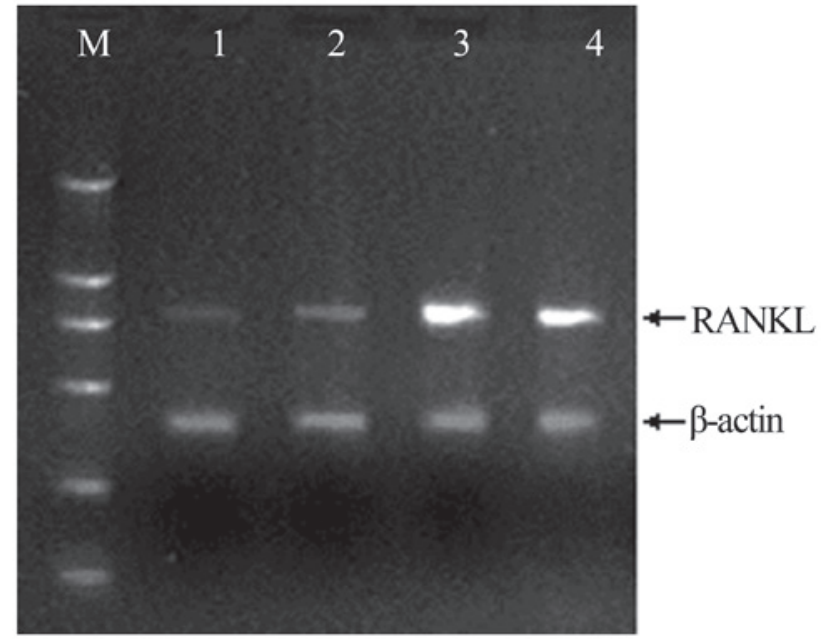

Figure 4. Expression of RANKL mRNA in rheumatoid pannus tissue. Lanes: $\mathrm{M}$, molecular weight standards (DL-2000); 1, control group; 2, collagen-induced group at 2 weeks; 3 , collagen-induced group at 4 weeks; 4 , collagen-induced group at 6 weeks. RANKL, receptor activator of nuclear factor $\kappa \mathrm{B}$ ligand.

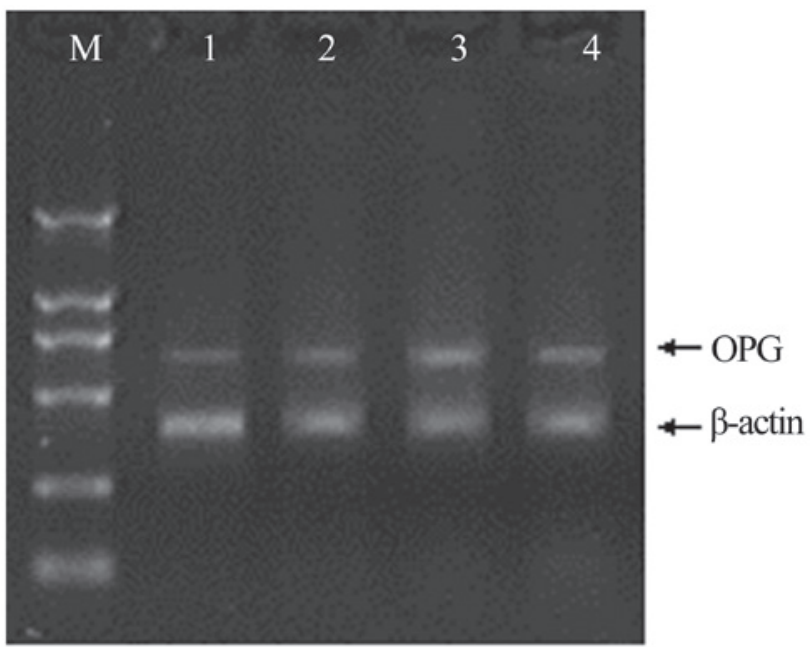

Figure 5. Expression of OPG mRNA in rheumatoid pannus tissue. Lanes: M, molecular weight standards (DL-2000); 1 , control group; 2 , collagen-induced group at 2 weeks; 3 , collagen-induced group at 4 weeks; 4 , collagen-induced group at 6 weeks. OPG, osteoprotegerin.

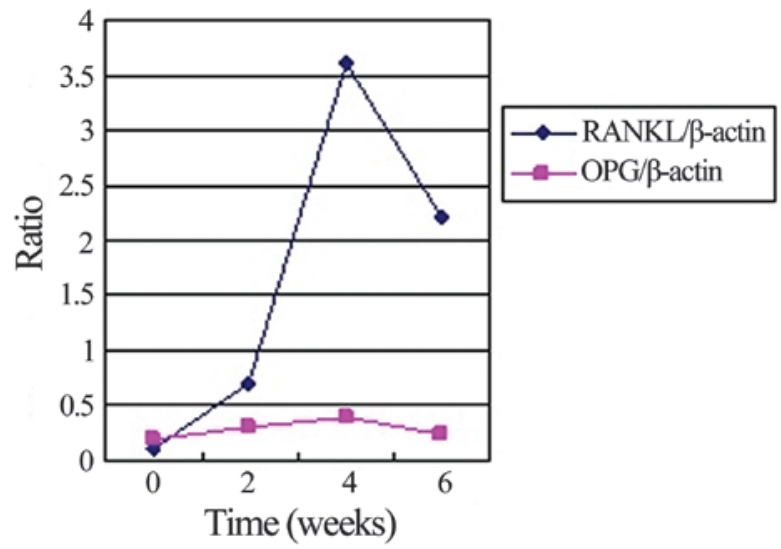

Figure 6. Ratio of RANKL: $\beta$-actin and OPG: $\beta$-actin expression in the condylar cartilage of joints with collagen-induced arthritis. Ratios were determined using the reverse transcription-polymerase chain reaction. RANKL, receptor activator of nuclear factor $\mathrm{\kappa B}$ ligand; $\mathrm{OPG}$, osteoprotegerin. 


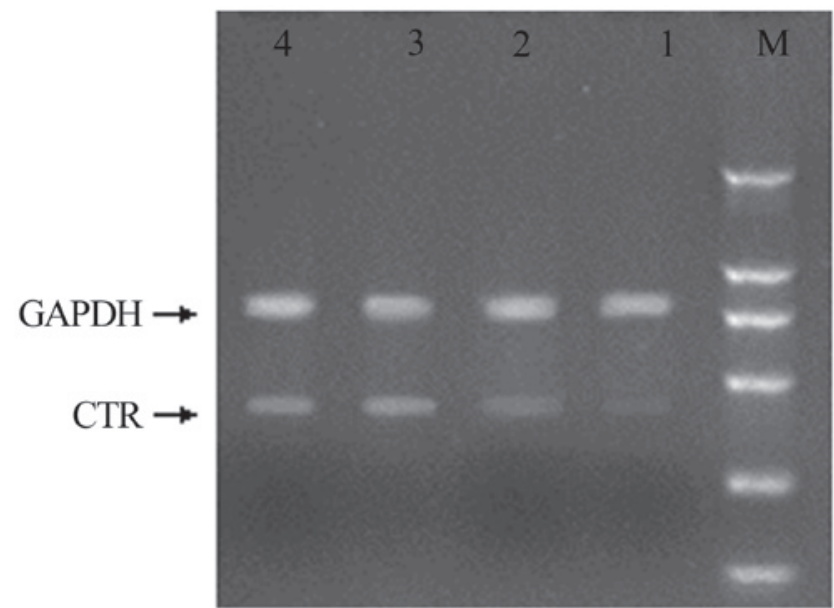

Figure 7. Expression of CTR mRNA in rheumatoid pannus tissue. Lanes: M, molecular weight standards (DL-2000); 1, control group; 2, collagen-induced group at 2 weeks; 3 , collagen-induced group at 4 weeks; 4, collagen-induced group at 6 weeks. CTR, calcitonin receptor.

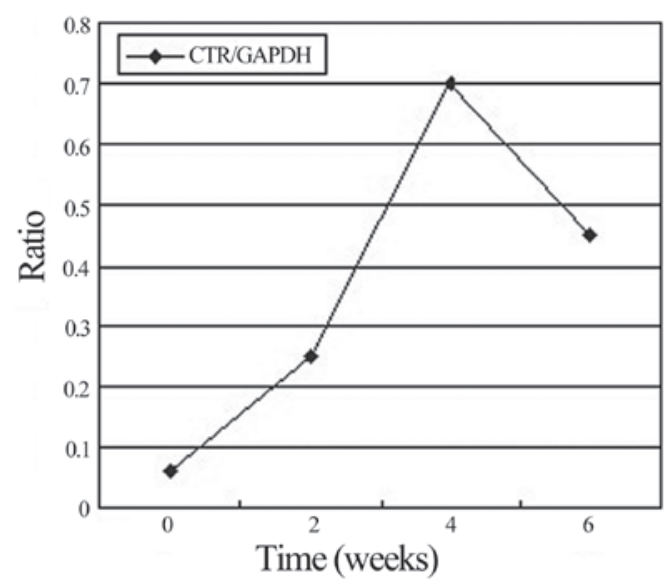

Figure 8. Ratio of CTR:GAPDH expression in the condylar cartilage of temporomandibular joints with collagen-induced arthritis. Ratios were determined using the reverse transcription-polymerase chain reaction. CTR, calcitonin receptor.

The mRNA expression levels of CTR peaked 4 weeks after immunization, and at weeks 2 and 6 they were significantly higher than the levels in the synovium of non-immunized rats (Fig. 7). Mirroring the increase in the number of osteoclasts, the level of CTR mRNA in the CTR-positive osteoclasts on the trabecular bone surface was 10.9- and 7.8-fold higher in rats with CIA at weeks 4 and 6 after challenge, respectively, than that in the control rats (Fig. 8).

\section{Discussion}

This study is the first, to the best of our knowledge, to demonstrate the changes in CTR-positive osteoclastic cells that develop in the mandibular cartilage of the TMJ during the progression of autoimmune inflammatory joint disease in the rat CIA model. The fact that the multinucleated cells found within erosive pits are osteoclasts can be confirmed by their expression of CTR. This finding in CIA is consistent with the findings in human RA (21), which suggests that CTR expression can be consistently found in rheumatoid tissues; osteoclasts readily form within these tissues, and this indicates that the expression of CTR is associated with the later stages of osteoclast differentiation. Bone erosion has been demonstrated to be correlated with CTR expression in long-term cultures of bone marrow, and CTR expression is considered to be a reliable marker in vitro and in vivo for the osteoclast phenotype (8).

Among the models of RA, the CIA model, which exhibits numerous morphological similarities to human RA, including pannus formation, patterns of synovitis and cartilage and bone erosion, has been the most widely studied; however, external clinical signs of erythema and induration in TMJ arthritis are generally not evident. These findings are in contrast to those of CIA of the knee joint, where swelling and erythema are frequently observed and used to grade the severity of arthritis $(22,23)$. The fact that TMJ swelling is rarely observed, in contrast to swelling of the knee joint, is likely due to the fact that the inflammatory exudate is able to be distributed into anatomical spaces medial, posterior and anterior to the TMJ, thus causing less external swelling.

From the observation of animal models, it has been suggested that RANKL plays a pivotal role in osteoclast generation and in the subsequent bone erosion in inflammatory arthritis (24). The present study clearly demonstrated a significant increase in RANKL levels and an increase in OPG levels in the TMJ of the CIA rats. It was found that the RANKL:OPG ratio was 9.0:1 and 6.4:1 in the CIA rats at weeks 4 and 6, respectively, which was considerably higher than the ratio in the control rats (1.0:2). These data therefore suggest a correlation between the RANKL:OPG expression ratio and the severity of RA in the TMJ. This finding is in agreement with the data of Geusens et al (15), which demonstrated that the radiographic progression of the bone component of joint destruction was dependent on osteoclast activation (i.e., the OPG:RANKL ratio) in animal models of arthritis. The present data demonstrated that CIA is associated with an imbalance in the RANKL:OPG system that favors RANKL; this imbalance occurs both locally, in foci of bone metabolism, and in the systemic circulation. The RANKL:OPG ratio is significantly higher in more severe cases of RA. Such an imbalance has already been considered for the osteoclast cells that modify the human bone marrow environment and induce osteoclastogenesis (20). With regard to the role of increased OPG in the affected TMJ, the marked increase of OPG in the synovial fluid may indicate that OPG contributes to the protection against further cartilage degradation or it may be reflective of a compensatory response by macrophages, chondrocytes or synovial fibroblasts to the destabilization of the normal balance between the degradation and synthesis of articular cartilage.

The RANKL:OPG expression ratio was significantly increased in the CIA rats compared with that in the control rats; as a result, there was an imbalance that favored RANKL and, therefore, bone resorption. We cannot, however, exclude the possibility of an alternative mechanism of osteoclast activation involving the release of growth factors from osteoblast-like or immune cells. In this way, the action of cytokines such as TNF- $\alpha$ and IL-1 $\beta$ on bone cells would be independent of RANKL and would result in the RANK- and 
OPG-independent modulation of osteoclast activity (25). In the present study, the serum levels of TNF- $\alpha$ and IL- $1 \beta$ during the acute phase were found to be at increased levels; these cytokines have been found to be associated with inflammatory diseases such as RA that produce localized bone loss $(18,26)$. A positive correlation was noted between all the inflammatory cytokines and RANKL, as well as CTR, the marker for osteoclasts, which indicated that these cytokines were involved in the differentiation of osteoclasts.

The present study has provided the first evidence, to the best of our knowledge, that the focal bone destruction occurring in experimental TMJ arthritis is attributable to cells exhibiting defining characteristics of osteoclasts, including CTR expression. The RANKL and OPG mRNA expression within the inflamed synovium has given an insight into the mechanism underlying the differentiation and function of osteoclasts at the sites of bone erosion in arthritis. OPG and/or agents targeting RANKL should be considered as novel therapeutic strategies to attenuate the bone loss and internal derangement of the TMJ in RA.

\section{References}

1. Scolozzi P, Bosson G and Jaques B: Severe isolated temporomandibular joint involvement in juvenile idiopathic arthritis. J Oral Maxillofac Surg 63: 1368-1371, 2005.

2. Lin YC, Hsu ML, Yang JS, Liang TH, Chou SL and Lin HY: Temporomandibular joint disorders in patients with rheumatoid arthritis. J Chin Med Assoc 70: 527-534, 2007.

3. Helenius LM, Tervahartiala P, Helenius I, et al: Clinical, radiographic and MRI findings of the temporomandibular joint in patients with different rheumatic diseases. Int J Oral Maxillofac Surg 35: 983-989, 2006.

4. Narváez JA, Narváez J, Roca Y and Aguilera C: MR imaging assessment of clinical problems in rheumatoid arthritis. Eur Radiol 12: 1819-1828, 2002.

5. Quinn JH: Arthroscopic and histologic evidence of chondromalacia in the temporomandibular joint. Oral Surg Oral Med Oral Pathol 70: 387-392, 1990.

6. Kapila S, Lee C, Tavakkoli Jou MR, Miller AJ and Richards AW: Development and histologic characterizations of an animal model of antigen-induced arthritis of the juvenile rabbit temporomandibular joint. J Dent Res 74: 1870-1879, 1995.

7. Horton MA, Lewis D, McNulty K, Pringle JA and Chambers TJ: Monoclonal antibodies to osteoclastomas (giant cell bone tumors): Definition of osteoclast-specific cellular antigens. Cancer Res 45: 5663-5669, 1985.

8. Quinn JM, Morfis M, Lam MH, et al: Calcitonin receptor antibodies in the identification of osteoclasts. Bone 25: 1-8, 1999.

9. Roodman GD: Advances in bone biology: The osteoclast. Endocr Rev 17: 308-332, 1996.

10. Romas E, Bakharevski O, Hards DK, Kartsogiannis V, Quinn JM, Ryan PF, Martin TJ and Gillespie MT: Expression of osteoclast differentiation factor at sites of bone erosion in collagen-induced arthritis. Arthritis Rheum 43: 821-826, 2000.
11. Hofbauer LC and Heufelder AE: Role of receptor activator of nuclear factor-kappaB ligand and osteoprotegerin in bone cell biology. J Mol Med (Berl) 79: 243-253, 2001.

12. Ohazama A, Courtney JM and Sharpe PT: Opg, Rank and Rankl in tooth development: Co-ordination of odontogenesis and osteogenesis. J Dent Res 83: 241-244, 2004.

13. Wakita T, Mogi M, Kurita K, Kuzushima M and Togari A: Increase in RANKL: OPG ratio in synovia of patients with temporomandibular joint disorder. J Dent Res 85: 627-632, 2006.

14. Haynes DR1, Crotti TN, Loric M, Bain GI, Atkins GJ and Findlay DM: Osteoprotegerin and receptor activator of nuclear factor kappaB ligand (RANKL) regulate osteoclast formation bycells in the human rheumatoid arthritic joint. Rheumatology (Oxford) 40: 623-630, 2001.

15. Geusens PP, Landewé RB, Garnero P, et al: The ratio of circulating osteoprotegerin to RANKL in early rheumatoid arthritis predicts later joint destruction. Arthritis Rheum 54: 1772-1777, 2006.

16. Martínez-Calatrava MJ,Prieto-Potín I, Roman-Blas JA, Tardio L, Largo R and Herrero-Beaumont G: RANKL synthesized by articular chondrocytes contributes to juxta-articular bone loss in chronic arthritis. Arthritis Res Ther 18: R149, 2012.

17. Brand DD, Latham KA and Rosloniec EF: Collagen-induced arthritis. Nat Protoc 2: 1269-1275, 2007.

18. Hirota Y, Habu M, Tominaga K, et al: Relationship between TNF-alpha and TUNEL-positive chondrocytes in antigen-induced arthritis of the rabbit temporomandibular joint. J Oral Pathol Med 35: 91-98, 2006.

19. Matsukawa A, Ohkawara S, Maeda T, Takagi K and Yoshinaga M: Production of IL-1 and IL-1 receptor antagonist and the pathological significance in lipopolysaccharide-induced arthritis in rabbits. Clin Exp Immunol 93: 206-211, 1993.

20. Grimaud E, Soubigou L, Couillaud S, et al: Receptor activator of nuclear factor kappaB ligand (RANKL)/osteoprotegerin (OPG) ratio is increased in severe osteolysis. Am J Pathol 163: 2021-2031, 2003.

21. Gravallese EM, Harada Y, Wang JT, Gorn AH, Thornhill TS and Goldring SR: Identification of cell types responsible for bone resorption in rheumatoid arthritis and juvenile rheumatoid arthritis. Am J Pathol 152: 943-951, 1998.

22. Kuruvilla AP, Shah R, Hochwald GM, Liggitt HD, Palladino MA and Thorbecke GJ: Protective effect of transforming growth factor beta 1 on experimental autoimmune diseases in mice. Proc Natl Acad Sci USA 88: 2918-2921, 1991.

23. Holmdahl R, Jansson L, Andersson M and Jonsson R: Genetic, hormonal and behavioural influence on spontaneously developing arthritis in normal mice. Clin Exp Immunol 88: 467-472, 1992.

24. Boyce BF and Xing L: Functions of RANKL/RANK/OPG in bone modeling and remodeling. Arch Biochem Biophys 473: 139-146, 2008.

25. Kwan Tat S, Padrines M, Théoleyre S, Heymann D and Fortun Y: IL-6, RANKL, TNF-alpha/IL-1: Interrelations in bone resorption pathophysiology. Cytokine Growth Factor Rev 15: 49-60, 2004.

26. Tominaga K, Alstergren P, Kurita H, Matsukawa A, Fukuda J and Kopp S: Interleukin-1beta in antigen-induced arthritis of the rabbit temporomandibular joint. Arch Oral Biol 46: 539-544, 2001. 